\title{
Modelo de gestión de marketing para el turismo rural comunitario
}

\section{Marketing management model for community rural tourism}

1 Erika Melany Lema Gallardo https://orcid.org/0000-0002-0124-6104 Pontificia Universidad Católica del Ecuador, Maestría en Gestión de Empresas Turísticas. Ambato, Ecuador eri.lema.gallardo@gmail.com

2 Esther del Carmen Mullo Romero https://orcid.org/0000-0002-4013-261X Pontificia Universidad Católica del Ecuador, Maestría en Gestión de Empresas Turísticas. Ambato, Ecuador emullo@pucesa.edu.ec

Artículo de Investigación Científica y Tecnológica Enviado: 07/11/2021

Revisado: 22/11/2021

Aceptado: 08/12/2021

Publicado:05/01/2022

DOI: https://doi.org/10.33262/visionariodigital.v6i1.1958

Lema Gallardo, E. M., \& Mullo Romero, E. del C. (2022). Modelo de gestión de marketing Cítese: para el turismo rural comunitario. Visionario Digital, 6(1), 25-49. https://doi.org/10.33262/visionariodigital.v6i1.1958

VISIONARIO DIGITAL, es una Revista Científica, Trimestral, que se publicará en soporte electrónico tiene como misión contribuir a la formación de profesionales competentes con visión humanística y crítica que sean capaces de exponer sus resultados investigativos y científicos en la misma medida que se promueva mediante su intervención cambios positivos en la sociedad. https://visionariodigital.org

La revista es editada por la Editorial Ciencia Digital (Editorial de prestigio registrada en la Cámara Ecuatoriana de Libro con No de Afiliación 663) www.celibro.org.ec 


\section{Palabras}

claves: modelo

de gestión,

turismo rural comunitario,

marketing

turístico,

turismo

Riobamba,

tendencia

turística,

marketing,

administración.

\section{Keywords:}

management

model, rural community

tourism,

tourism

marketing,

Riobamba
Resumen

Introducción. Dentro del marco de las tendencias turísticas mundiales, el turismo comunitario muestra un interés progresivo en la industria debido al enfoque conservacionista de la biodiversidad, la cultura y tradiciones de una localidad, además de generar divisas y mejorar la calidad de vida de la comunidad y de sus habitantes en virtud de un producto turístico de calidad en donde el viajero y la comunidad obtengan beneficios. Objetivo. Sobre esta base, se plantea un modelo de gestión del marketing turístico rural fundamentado en la imagen del sitio, cuyo objetivo general es desarrollar un modelo de gestión de marketing para el turismo rural comunitario de Riobamba. Metodología. Para esto, se ha utilizado como metodología la investigación descriptiva/explicativa y se ha manejado técnicas de investigación bibliográfica, así como, también salidas de campo para la realización de encuestas a un nivel analítico y prospectivo, el mismo que en su complexión sigue los fundamentos de la participación, el cooperativismo y el compromiso de los partícipes que interactúan como vía para conseguir la calidad del producto. Resultados. En consecuencia, se espera preservar, aprovechar y desarrollar el destino turístico con una adecuada orientación estratégica de marketing en zonas rurales de Riobamba, destacando la importancia de la comunicación, que, gracias a la llegada del internet, ha hecho que surjan nuevas herramientas de difusión de información debido al aumento de la conectividad e interactividad en la sociedad. Conclusión. Sobre la base de este marco las autoridades de la ciudad de Riobamba pueden iniciar procesos de sensibilización del este artículo con la población con la intención de mejorar la situación económica de aquellas parroquias que, debido a la mala gestión, su potencial todavía no ha sido explotado.

\section{Abstract}

Introduction. Within the framework of world tourism trends, community tourism shows a progressive interest in the industry due to the conservationist approach to biodiversity, the culture and traditions of a locality, in addition to generating foreign exchange and improving the quality of life of the community and of its inhabitants by virtue of a quality tourism product where the traveler and the community obtain benefits. Objective. On this basis, a rural tourism marketing management model is proposed based on the 
tourism, tourism trend. image of the site, the general objective of which is to develop a marketing management model for rural community tourism in Riobamba. Methodology. For this, descriptive / explanatory research has been used as a methodology and bibliographic research techniques have been used, as well as field trips to carry out surveys at an analytical and prospective level, which in its complexion follows the fundamentals of participation, cooperatives and the commitment of the participants who interact to achieve product quality. Results. Consequently, it is expected to preserve, take advantage of and develop the tourist destination with an adequate strategic marketing orientation in rural areas of Riobamba, highlighting the importance of communication, which, thanks to the arrival of the internet, has led to the emergence of new dissemination tools. of information due to the increase in connectivity and interactivity in society. Conclusion. Based on this framework, the authorities of the city of Riobamba can initiate awareness-raising processes of this article with the population with the intention of improving the economic situation of those parishes that, due to mismanagement, their potential has not yet been exploited.

\section{Introducción}

La actividad turística se ha convirtiendo en uno de los fenómenos económicos más importantes del siglo XXI según la Organización Mundial del Turismo ([OMT], 2021) caracterizado por la digitalización de las empresas del sector turístico y el comienzo de una tendencia relacionada con la sostenibilidad, la experiencia vivencial, la desconexión y nuevas estilos de turismo en destinos pocos aglomerados; el turismo, tiene la potencialidad de promover el desarrollo mercantil, lo cual a su vez se traduce en oportunidades de empleo, de conservación, de concientización y en impulso de otras actividades comerciales del sector primario dedicadas a la producción de bienes, como actividades agropecuarias, de restauración, y del sector secundario, encaminadas a la transformación de bienes, como la industria textil, las artesanías y el transporte.

Es así como, de acuerdo con el Ministerio de Turismo ([MINTUR], 2020) el turismo ha contribuido con el 2,2\% al Producto Interno Bruto (PIB) del Ecuador en el 2019, esto, debido al interés de los viajeros sobre el particular modelo de turismo que brinda el Ecuador, un turismo sostenible, basado en los 4 ejes fundamentales: económico, ambiental, social y cultural. 
En tal virtud, la oferta turística del Ecuador es diversa, debido a sus: condiciones geográficas, climáticas, cuatro regiones: Costa, Sierra, Oriente y Galápagos, biodiversidad y riqueza social y cultural con 14 nacionalidades y 18 pueblos, con su propia cosmovisión, integrada a una gran variedad de destinos turísticos reconocidos por la Organización de las Naciones Unidas para la Educación, la Ciencia y la Cultura ([UNESCO], 2021) como patrimonios culturales, y numerosos sitios de gran belleza natural, ha dado lugar a que en las últimas décadas, las comunidades iniciaran en la participación de actividades turísticas, con un concepto propio: el turismo rural comunitario.

Así pues, el Turismo Rural Comunitario (TRC) busca desarrollar la actividad turística de manera planificada y sostenible y se basa en la participación de las comunidades rurales, campesinas, indígenas, mestizas o afrodescendientes, y así generar ingresos complementarios a las actividades económicas diarias que se desarrollan en la colectividad y conservar los recursos del territorio.

Es así como, el TRC nace en Ecuador hace 35 años en las Comunidades Organización de los Pueblos Indígenas del Pastaza (OPIP) con proyectos exitosos desarrollados por la Federación Plurinacional de Turismo Comunitario del Ecuador (FEPTEC) y que en la actualidad se mantienen en crecimiento continuo. Por el contrario, en el cantón Riobamba, provincia de Chimborazo, las oportunidades y circunstancias no han sido aprovechadas.

Así mismo, el TRC reúne diversos modelos de gestión, que tienen como principal elemento la participación de la localidad en la actividad turística de forma sostenible. Uno de ellos es un modelo de gestión de marketing, ya que es una herramienta eficaz para contribuir a la difusión de los destinos, al ser un instrumento de desarrollo e inserción sostenible, para que los posibles clientes se sientan atraídos. Es así como, como estrategia de mercado, se busca desarrollar marcas turísticas que reconozcan los atractivos de un lugar y potenciarlos al máximo para posesionarlos como una marca.

A partir de lo expresado, se describe la situación problémica: en la actualidad, un gran número de Centros de Turismo Comunitario (CTC) de las parroquias rurales de Riobamba se encuentran inoperantes o no han podido certificarse o legalizarse, en su mayoría, debido a que no solventan un flujo suficiente de visitantes primordiales para la sostenibilidad del proyecto, a causa de la escasa difusión del turismo rural comunitario. A partir de lo indicado, se formula el problema científico: ¿Cómo mejorar la gestión efectiva y proactiva del turismo rural comunitario?

Para dar solución a lo planteado, la hipótesis de esta investigación es: el modelo de gestión de marketing mejora la difusión el turismo rural comunitario de Riobamba. De tal manera que, el objetivo general de esta investigación es desarrollar un modelo de gestión de 
marketing para el turismo rural comunitario. De manera que los objetivos específicos son: Construir el marco teórico-referencial a partir de un análisis crítico en temáticas vinculadas con el turismo rural comunitario y la dinámica del marketing; identificar las herramientas y procedimientos metodológicas para la creación del modelo de gestión de marketing del turismo rural comunitario y; diseñar el modelo de gestión de marketing para el turismo rural comunitario de Riobamba.

Por esta razón, se ha utilizado como metodología la investigación descriptiva/explicativa y se ha manejado técnicas de investigación bibliográfica, así como también salidas de campo para la realización de encuestas a un nivel analítico y prospectivo, el mismo que en su complexión sigue los fundamentos de la participación, el cooperativismo y el compromiso de los partícipes que interactúan como vía para conseguir la calidad del producto.

Con respecto a la justificación, esta investigación pretende generar datos confiables sobre la oferta y la demanda turística de las comunidades rurales del cantón Riobamba, los mismos que permitan la futura implementación de proyectos de gestión turística adecuada y con ello, al desarrollo turístico sostenible de las comunidades, creando propuestas de marketing que engloben al turismo como eje productivo vinculado con la parte comercial $\mathrm{y}$, visualicen al cantón Riobamba, con todas sus parroquias rurales como una alternativa turística.

\section{Modelo de Gestión}

Existen distintas definiciones de modelo, pero una de las más acertadas es la de Torres (2016), en donde manifiesta que un modelo es una instrumento que permite analizar, describir, representar los datos que se obtienen en un proceso para obtener información, con el objetivo de tomar la mejor decisión, sin olvidar el acompañamiento de una efectiva gestión administrativa. Del mismo modo, para Patiño (2016) se establece que un modelo es una descripción resumida de una realidad anhelada. Es así como un modelo turístico establece los patrones, indicadores y ejes para orientar el dinamismo en un territorio.

En base a lo que manifiesta Torres y Patiño, el enfoque primordial de un modelo es un diseño completo de una determinada situación ideal, en donde se prescribe paso a paso el camino a seguir para la adecuada toma de decisiones por parte de un gestor a cargo.

Es probable que con la diversidad de modelos que existen y la realidad propia de cada lugar, con todas las variables de por medio (ubicación geográfica, costumbres, capital, etc.), no figure ningún concepto o modelo dominante, de ahí que los investigadores continúan explorando diferentes caminos. En efecto, es importante señalar que tanto los investigadores como los gestores de destinos reconozcan que existe esta complejidad y que consideren minuciosamente el modelo elegido, sin olvidar que las características en 
las que todo modelos se basa son: la problemática analizada, el objetivo de la gestión, los actores principales, las prioridades y las posibles vías para tener soluciones eficientes y eficaces (Pearce, 2016).

Así mismo, para García (2017) existen innumerables tipos de modelos de gestión de destinos que reflejan una realidad turística, cada modelo tiene sus fortalezas y limitaciones, ningún modelo es completo o dominante, de forma que la nueva tendencia es una adecuada mixtura o integración entre dos o más de modelos. De igual manera, la diversidad de modelos que existen según Pearce (2016) esclarece que no hay conceptos y modelos dominantes, por lo que es preciso examinar cual es el modelo más adecuado para cada caso, adaptándolo a la realidad de cada territorio.

Por su parte, un modelo de gestión es una guía de referencia para la administración de una organización, tanto en las empresas privadas como en la administración pública. Asimismo, en un modelo de gestión se establecen políticas y acciones para alcanzar objetivos, y, la necesidad de potenciar esta gestión en instalaciones turísticas con el fin de brindar un producto que se apropie a las expectativas de un cliente que cada vez es más exigente. Por el contrario, modelo de gestión del ámbito privado es diferente el modelo de gestión que utilizan las organizaciones públicas, en concreto, el primero busca la obtención de ganancias económicas, y el segundo aspira al bienestar social de la localidad (Valdiviezo, 2015).

De la misma manera, los gobiernos tienen varios modelos de gestión que se fundamentan en el desarrollo de acciones y políticas, y con el cual pretenden alcanzar sus objetivos. En este sentido, un modelo de gestión en una comunidad promete el desarrollo de un servicio turístico de calidad por parte de la participación de la comunidad de manera controlada y ordenada en la planificación y ejecución de operaciones que beneficien a potenciar el progreso sostenible de la productos y servicios procedentes de la actividad turística.

En conjunto García (2020) señala que el modelo de gestión turística debe manejar adecuadamente el turismo sostenible mediante la planificación y organización del trabajo de forma metodológica, es necesario además realizar un diagnóstico del área y realizar un estudio profundo para obtener las mejores estrategias, con el fin de aplicarlas en los distintos ejes de la comunidad, de forma sostenible en cada proceso de planificación estratégica, adaptándose al ámbito del territorio, contribuyendo al desarrollo del conocimiento de la actividad turística.

Cabanilla (2018) destaca la utilidad en la aplicación de un modelo de gestión en una empresa social, ya que afirma que el propósito principal no es el beneficio económico de los accionistas, sino la generación de impactos positivos para todas las partes de una comunidad. 
Así, por ejemplo: "un modelo de gestión de los recursos desde la perspectiva de protección y conservación de los recursos para el aprovechamiento de futuras generaciones, es necesario llevar a cabo un modelo de planificación" (Santamaría et al., 2017, p. 221).

De la misma forma, la metodología de un modelo de gestión propuesto por el MINTUR (2020) define parámetros de organización, administración, funcionamiento, operación, mantenimiento y actualización de las fases de implementación, los mecanismos de control y la normativa actual aplicable para las partes, cuyas facilidades y equipamientos se construyan con recursos fiscales. Así también, hay que tener en cuenta las tarifas, los mecanismos de promoción y difusión, de los productos y servicios a ofertarse.

Así mismo López et al. (2015) proponen un modelo híbrido compuesto por el Sistema de Información de Mercadotécnica (SIM) y el sistema de apoyo a las decisiones de marketing, el cual lo conforman tres subsistemas: investigación, inteligencia y registro interno, así:

1. Investigación: es el sondeo concerniente con los consumidores dependientes y potenciales

2. Inteligencia: son los datos del medio externo y

3. Registro interno: es la información derivada de ejecutar dinamismos de mercadeo al interior de la organización.

Según CELTA (2018) para implementar un modelo de gestión turística, es fundamental considerar el patrimonio que posee el lugar, ya que funciona como factores de atracción y marca la diferencia con la competencia, estos atractivos pueden ser aprovechados en la gestión de marketing y publicidad para definir la imagen de un determinado centro turístico.

En otros términos, el modelo busca ser una herramienta de análisis para impulsar la gestión turística comunitaria rural a través del GAD Parroquial de Riobamba, con el objetivo de mejorar las condiciones socioeconómicas del sector, teniendo en consideración al turismo como una fuente complementaria de ingresos (Torres, 2016).

\section{Marketing Turístico}

Hoy en día, el marketing turístico contribuye a la economía de los países por medio del interés que genera en los turistas por visitar los atractivos que dicho país posea. Es así como en un mundo cada día más dinámico y en constante evolución, se considera a la información un recurso indispensable para el desarrollo de las diligencias de las organizaciones. La información constituye el punto de partida del marketing (Ramírez \& Perusquia, 2019). 
En relación con lo que Ramírez y Perusquia manifiestan, conviene subrayar que, sin la correcta recolección de información, cualquier sistema de marketing fracasaría, tanto de la demanda como de la oferta ya que el marketing busca hacer un "match" entre lo que el cliente quiere, versus lo que la oferta tiene para entregar.

De ahí que, un plan de marketing es un instrumento que beneficia a una empresa, ya que define dos de las características más importantes de un proceso comercial triunfante: el foco de lo que debe hacer y la claridad de propósito (Hoyos, 2016). Así pues, uno de los objetivos más importantes de toda empresa es el beneficio, y no solamente se refiere a "incrementar el volumen de ventas", sino que también se busca la satisfacción y bienestar del cliente, mediante las herramientas de que dispone la dirección comercial, ideando un marketing adecuado al mercado al que se quiera dirigir.

Para Mogrovejo et al. (2019) el marketing al tener cualidades estratégicas es una técnica sistemática de análisis y comprensión del mercado, con el afán de descubrir situaciones favorables que ayuden a la organización a cubrir las necesidades y superar las expectativas de los clientes de una forma eficiente y óptima, que el resto de competidores. En este sentido se analiza el consumidor, el mercado, posicionamiento del producto y la promoción y las estrategias las mismas.

Gracias al cambio de mentalidad con respecto a la utilidad del marketing, hoy en día las empresas ven el marketing como una herramienta para su negocio, y no como un gasto superfluo o un lujo. Financiar en marketing es invertir en el negocio, ya sea que la empresa aspire réditos económicos o no. El marketing puede dar mayor reconocimiento a la marca de una empresa o un negocio, ayuda a ganar o fidelizar clientes ya que promueve una mejor comunicación, los identificar, los conoce y así se obtiene información de primera mano sobre el perfil de nuestros clientes. Sin mencionar el ahorro de tiempo y dinero que el marketing ostenta (Valdiviezo, 2015).

Por otra parte, el estudio de mercado es el punto de partida para el marketing mix o la mezcla de mercadotecnia, conocida también como las 4P: producto, precio, plaza y promoción, que no son sino estrategias para incrementar la participación en el mercado y lograr un buen posicionamiento. En primera instancia para una correcta investigación de mercado se debe determinar qué productos o servicios se quiere alcanzar, posteriormente es necesario recabar información de a quién se prestará un servicio o se venderá un producto: conocer como son esos clientes, su localidad, de qué sectores económicos provienen, su grado de instrucción, su edad, su género. Una vez se tenga el perfil del cliente, a continuación, es importante visualizar cuáles son los mercados meta, es decir, a donde queremos llegar, entender la situación actual del mercado, saber el segmento a los que se desea llegar, para finalmente desarrollar una estrategia eficaz de promoción, capaz de satisfacer las necesidades tanto del demandante como del ofertante y así proceder con la descripción general de la cobertura de mercado (Vaca, 2015). 
En cuanto a "el plan de marketing" es un material de gestión por el cual se establecen los pasos a seguir, los tiempos y las técnicas o procesos para conseguir los objetivos de la empresa. Si bien un plan de marketing requiere de mucho tiempo, al final nos permitirá ahorrar tiempo, y concentrar esfuerzos.

Así pues, Vaca (2015) refiere dos tipos de planes de marketing clasificados en distintas categorías, según los objetivos:

1. Plan para un nuevo producto: Se realizan para productos, línea de productos, servicios, o marcas de la empresa que no han sido aún lanzadas.

2. Plan de marketing anual: Cuando se realiza para aquellos productos, proyectos, servicios o marcas ya establecidos en la línea de producción de una empresa. Estos planes deben ser inspeccionados periódicamente, de forma semestral o anual.

Por consecuente, es un sistema de carácter social por el cual las empresas buscan generar valor agregado a los productos o servicios, con el fin de cubrir las necesidades de un grupo de personas pertenecientes a un mercado (Vera, 2017). Al mismo tiempo que atrae a los turistas, les entrega servicios o productos de calidad para satisfacer y superar sus expectativas y así crear un entorno que favorece el desarrollo del destino (Pearce, 2016).

Considerando que para Guillén \& Vera (2020) la gestión del marketing es el arte y la ciencia de descubrir los mercados objetivos cualificados y crear relaciones rentables con los mismos. Para una mejor eficiencia del marketing es necesario precisar cuáles son las características y expectativas del cliente objetivo y preparar una estrategia que establezca una fidelización rentable entre la organización y el cliente. En la actualidad, tanto las estrategias de marketing como los gustos y las necesidades de los clientes y su entorno han cambiado, dejando de lado aquellos criterios que en primera instancia daban prioridad a la reducción de costos en la producción, es así como ahora las organizaciones se han adaptado hacia su consumidor, para establecer una gestión que haga énfasis en el servicio o producto y permita establecer relaciones mutuamente valiosas.

En particular, para el desarrollo del sector turístico es recomendable plantear esfuerzos de marketing innovadores sobre los atributos materialistas de gasto durante los viajes, pensar en cómo los viajes innovadores podrían representar el bienestar, la comodidad y la tranquilidad personal en un viaje experiencial favorable para los turistas, los cuales se sienten motivados a consumir bienes y servicios fuera de lo común (Chan et al., 2016).

Por lo tanto, la originalidad de marca de las actividades turísticas es una forma efectiva de atraer a estos turistas. Las campañas de difusión y promoción deben proyectar un valor agregado de los viajes, debe ser innovador para de esta manera tener una respuesta positiva de satisfacción por parte del turista. Se sugiere a los especialistas en marketing que transmitan una sensación de placer y disfrute. 
De la misma forma, el marketing social contribuye a una estrategia de comunicaciones unificadas que puede funcionar en todos los medios y elementos promocionales tales como: un mensaje, un lema, un logotipo y un enfoque de posicionamiento social, mutuamente, estas actividades regularizadas favorecen el acrecentamiento de la efectividad tal como se concibe a través de la fase de respuesta (Martin Key \& Czaplewski, 2017, citado por Saltos et al., 2018).

Conviene enfatizar que el marketing en las comunidades organizadas dedicadas al desarrollo del turismo local no cuentan con los recursos financieros suficientes para desarrollar una campaña de marketing a gran escala para publicitar sus recursos y atractivos turísticos que poseen como comunidad (Lucas-Marcillo, 2019). Por consiguiente, el presente modelo busca la optimización de recursos y la eficacia en los procesos adaptado a la realidad.

\section{Turismo rural comunitario}

Vera (2018) define que "El turismo se entiende como una actividad multidisciplinar que involucra el traslado temporal de individuos o grupos desde su lugar de residencia habitual hacia determinado sitio de interés turístico, tal traslado implica un gasto" (p. 11).

En cuanto al modelo turístico actual, desde una perspectiva generalista, está encaminado hacia una planificación y dirección responsable, con productos de calidad, los cuales, lejos de poner en peligro la conservación de los recursos de la zona contribuyen a considerarlos como la base primordial en la cual se asienta la actividad turística (Martínez et al., 2018). Es así, como refiere Reyes et al. (2017) el turismo a nivel mundial ha evolucionado en su enfoque a la sostenibilidad, evidencia un creciente interés hacia las áreas rurales, por sus tradiciones, su cultura, la tranquilidad y biodiversidad; particularidades que deben ser salvaguardadas para su correcto aprovechamiento a través de una apropiada instrucción estratégica.

Se quiere decir con esto que la nueva tendencia turística está enfocada en sostenibilidad, la cual, como principio básico, busca la protección del medio natural y cultural de un determinado lugar, garantizando réditos económicos, he ahí donde nace el turismo comunitario.

En pocas palabras, el turismo comunitario deriva de la iniciativa de la comunidad de revelar y expresar la esencia de su vida cotidiana, su cosmovisión, su cultura, la realidad de un día a día que transcurre diferente y a veces indiferente a este mundo globalizado (Rodas \& Sanmartín, 2015), y sin lugar a dudas como afirma Reyes et al. (2017) dentro del marco de las tendencias turísticas a nivel mundial el turismo comunitario es uno de los que alcanza mayor acogida en el mercado turístico.

Conviene subrayar que, el desarrollo de la actividad turística debe responder a una planificación íntegra, que considere como actor principal a la localidad, trabajando dentro 
de parámetros de equidad para la obtención de un beneficio común, capaz de generar un efecto multiplicador (Vera, 2018). De este modo, el turismo rural comunitario se enfoca en la formulación de propuestas turísticas que generen beneficio de forma equitativa a los actores y gestores de la localidad, mediante la toma de decisiones en un marco de respeto y acuerdo comunitario cuyos principios sean la inclusión e integración de los miembros de la comunidad (Campbell, 1999; Stronza \& Gordillo, 2008; ZizumboVillareal, 2013; Pérez, 2004, citado por Parra Cárdenas et al., 2019).

El turismo, al jactarse de sus bondades económicas y de generación de empleo, parece no considerar que el turismo comunitario está siendo muy cuestionado, debido a la escasa información sobre los resultados adquiridos y su competitividad en el mercado mundial (Alcívar, 2020).

Para García (2020) el turismo rural debe superar una serie de dificultades, como lo es la infraestructura (servicios básicos, agua potable y entubada, electricidad, caminos, comunicaciones, recolección de basura, hospitales y equipos médicos, policías y bomberos) que por lo regular es insuficiente, de mala calidad o inexistente.

En efecto, la comercialización de centros turísticos comunitarios es uno de los puntos a desarrollar, iniciando por la misma estructuración de los productos. La aplicación de técnicas adecuadas es fundamental tanto para el diseño de productos o servicios como para su comercialización si se quiere mantener la estabilidad del emprendimiento (Doumet \& Huerta, 2017).

Dicho de otra manera, el autor refiere que el turismo comunitario busca un intercambio intercultural de calidad para el visitante, ofreciendo sus recursos patrimoniales comunitarios a cambio de la mejora de la economía de la comunidad con el fin de prevalecer su cultura y todo lo que esto conlleva: su vestimenta, sus costumbres, su gastronomía, etcétera.

De manera que la demanda del turismo comunitario no solo está creciendo en el mercado internacional, sino que también la clientela nacional y local está suponiendo un fuerte factor de activación (Cañada, 2015). Según Guillén (2016) el turismo comunitario visto como un desarrollo socioeconómico en las zonas menos conferidas de recursos financieros, donde los comuneros buscan vías alternas de ingresos para su familia, representa una alternativa muy importante para las zonas rurales del Ecuador.

Por lo que se refiere al turismo comunitario en el Ecuador, se tiene registro que surgió a mediados de los años ochenta, son cerca de 231 emprendimientos que se han instaurado en el país, sin embargo, tan solo 99 se hallan activos al 2015 (Cabanilla et al., 2017).

Así pues, en Ecuador, en los últimos 30 años ha sobresalido como un país pionero de turismo comunitario en América Latina, puesto que desde la década de los 80, esta actividad se ha vuelto transcendental en el desarrollo del turismo nacional, muchas 
comunidades han previsto el valor de preservar el patrimonio, sus recursos y atractivos para las generaciones futuras, a la vez que éstos les generan beneficios en distintos campos de acción (Vera, 2018, p. 25).

Según Rodas \& Sanmartín (2015) la Federación Plurinacional de Turismo Comunitario del Ecuador (FEPTCE), impulsó la misión de fortalecer y posicionar el turismo comunitario del Ecuador dentro y fuera de las fronteras nacionales. Ahora bien, el MINTUR ha desarrollado el programa "Consolida Turismo Comunitario", que tiene por objeto mejorar el producto turístico comunitario a través del apoyo a las comunidades en asistencia técnica, capacitación, calidad y promoción; no obstante, para ser beneficiarios del programa, la comunidad debe estar legalmente reconocida tanto por la Secretaría de Pueblos y Nacionalidades como por el MINTUR.

Para Rodas \& Sanmartín (2015) cada comunidad es singular, con sus principios, valores, normas e instituciones que rigen su forma particular de organización y convivencia y el cantón Riobamba no es la excepción. Ubicada en la provincia de Chimborazo, San Pedro de Riobamba "La Sultana de los Andes" es un cantón con 225.741 habitantes aproximadamente según Centro Iberoamericano de Desarrollo Estratégico Urbano ([CIDEU], 2020), rodeada de hermosos paisajes, 5 parroquias urbanas y 11 parroquias rurales, lo hace un punto estratégico para el desarrollo del TRC que ha sido muy poco aprovechado.

\section{Metodología}

Se ha utilizado como metodología la investigación descriptiva/explicativa, pues se busca no sólo describir el problema, sino que también descubrir las causas del mismo referente al turismo rural comunitario. El estudio se ha desarrollado en 3 de las 11 parroquias rurales del cantón Riobamba de la provincia de Chimborazo: Cacha, Calpi y San Juan, ya que únicamente de estas 3 se conoce su labor en el turismo comunitario.

De este modo, para la compilación de información se ha manejado técnicas de investigación bibliográfica como artículos científicos, proyectos de investigación, reportes certificados por la Dirección de Gestión de Turismo del GADM de Riobamba, así como también salidas de campo para la observación científica de hechos y fenómenos que han sido registrados para su análisis posterior, y la recaudación de información de primera mano con el uso de la encuesta como técnica y el cuestionario como instrumento elaborado en la plataforma digital Google Forms, dado el volumen y el tipo de información cualitativa y cuantitativa que se proyecta obtener a un nivel analítico y prospectivo, el cual, en su complexión sigue los fundamentos de la participación, el cooperativismo y el compromiso de los partícipes que interactúan como vía para alcanzar la validez y veracidad de la investigación de siguiente manera: 
- Encuestas dirigidas a los ofertantes del servicio turístico rural comunitario, específicamente a los directivos y presidentes de las juntas comunitarias de las 3 parroquias:

- En Calpi: Palacio Real, Jatari Campesino, La Moya, Quilla Pacari

- En San Juan: Chakana, Casa Cóndor, Tambohuasha y

- En Cacha: Pucará Tambo

- Encuestas dirigidas a los demandantes de productos y servicios turísticos de la ciudad de Riobamba, para esto, se inicia con la segmentación de mercado. La primera segmentación considera una ubicación geográfica cercana, es decir, la población de la ciudad de Riobamba y para la segunda, se consideran personas comprendidas dentro de la Población Económicamente Activa (PEA) con un rango de edades comprendidas entre los 18-65 años, con capacidad de gasto, así:

\section{Tabla 1}

\section{Segmento de mercado}

\begin{tabular}{lll}
\hline \multicolumn{1}{c}{ 1ra. Segmentación } & \multicolumn{1}{c}{ 2da. Segmentación } & Fuente \\
\hline Población & Hombres y mujeres económicamente & \\
Económicamente & activos por grupos principales de & \\
Activa de la ciudad de & $\begin{array}{l}\text { ocupación del sector urbano de la INEC } \\
\text { Riobamba. }\end{array}$ & $\begin{array}{l}\text { ciudad de Riobamba cuyas edades } \\
\text { están comprendidas entre 18 y } 65\end{array}$ \\
& años.
\end{tabular}

Fuente: Elaboración propia

Posteriormente, es necesario calcular el universo de estudio, el cual se refiere al total de la segmentación de mercado, multiplicado por el índice de la migración (1.03\%) a nivel nacional, en el año 2020.

\section{Tabla 2}

Universo de estudio

\begin{tabular}{lll}
\hline \multicolumn{1}{c}{ Segmentación } & \multicolumn{1}{c}{ Ciudad } & \multicolumn{1}{c}{ Fuente } \\
\cline { 2 - 3 } & Riobamba & \\
\hline Población Económicamente Activa de 197.940 & & Resultados del Censo de \\
la ciudad de Riobamba. & & Población y Vivienda del \\
& & $\begin{array}{l}\text { Ecuador 2010 realizado por } \\
\text { el Instituto Nacional de }\end{array}$ \\
& & $\begin{array}{l}\text { Estadísticas y Censos } \\
\text { (INEC) }\end{array}$ \\
\hline
\end{tabular}




\section{Tabla 2}

Universo de estudio (continuación)

\begin{tabular}{clc}
\hline Índice de la migración & $1,03 \%$ & Banco Central del Ecuador. \\
\hline UNIVERSO DE ESTUDIO & 178.269 & \\
\hline Fuente: Elaboración propia &
\end{tabular}

Consecutivamente, para determinar la muestra se aplicó la fórmula matemática de la muestra para poblaciones finitas de Canavos, obteniendo un total de 383 encuestas. Así, los cuestionarios se aplicaron por medios digitales las cuales tienen 21 preguntas divididas en 4 secciones: datos generales, modelo de gestión, aspecto turístico, aspecto de marketing.

Con respecto a la recopilación de la información, corresponde al uso de material bibliográfico y/o documental referente a libros, revistas, artículos, del mismo modo que se obtiene información virtual de páginas WEB para expandir, profundizar contextualizaciones y criterios fundamentales para el sustento del tema de investigación, cuyas fuentes específicas han sido: Instituto Nacional de Estadísticas y Censos (INEC), Banco Central del Ecuador (BCE). En efecto, para el trabajo de campo se ha utilizado como instrumento de recopilación de información la encuesta. Acto seguido, se ha sistematizado la información para así obtener el perfil del turista, que no es sino una recopilación de todas las preguntas de la encuesta, ordenadas y analizadas de manera que se pueda determinar cómo se piensa que sería el turista.

Para el cumplimiento del análisis de la oferta turística existente en las parroquias rurales del cantón Riobamba, Provincia de Chimborazo, se ha determinado la oferta actual de turismo comunitario referente a los recursos y facilidades turísticas que posee, para ello se ha establecido el inventario de atractivos turísticos naturales y culturales además del catastro del año en curso, así:

En primer lugar, para el levantamiento de la información referente a los atractivos naturales y culturales, se ha utilizado como técnica el fichaje y como instrumento las fichas, para ello se ha empleado la metodología para identificar atractivos turísticos aprobada por el MINTUR.

A continuación, para el catastro, al ser una herramienta referencial que contiene el resultado de la recopilación de los insumos utilizados dentro de los procesos para el registro y actualización de datos de las entidades legalmente patentadas en el MINTUR, han sido debidamente constatadas en las salidas de campo.

Finalmente, los datos proporcionados por la Información Turística (iTour), y otras fuentes secundarias como los sitios web del Gobierno Provincial de Chimborazo, de la Dirección 
de Gestión de Turismo de Riobamba, Riobamba Turismo, entre otras han sido confirmadas y evaluadas para obtener datos confiables y actualizados.

Así pues, con la información obtenida, se ha podido tener una base sólida para poder aplicar el mix de marketing: producto, precio, plaza y promoción, teniendo en cuenta los nuevos canales de desarrollo en la era digital, como lo son las redes sociales, considerando la caducidad que tiene hoy en día los medios publicitarios tradicionales como la televisión o radio.

\section{Resultados}

\section{Resultados de la demanda}

Considerando que el 35\% de los encuestados representan a la PEA con la edad entre los 31 y 40 años, mientras que el 4 y 5\% son entre los 61 y 70 años y 18 y 20 años respectivamente. Asimismo, el 54\% de los encuestados son de género femenino y el $46 \%$ masculino y el $77 \%$ de los encuestados residen en la ciudad de Riobamba, y un $4 \%$ que representa "Otros" en ciudades como: Tena, Pallatanga, Puyo, Mocha y Santa Elena, se obtuvieron los siguientes resultados:

\section{Aspecto modelo de gestión}

Por lo que refiere a Pearce (2016), señala que el manejo de modelos contribuye a la correcta identificación de los problemas claves y la utilización de tiempo y recursos con más eficacia. Es otras palabras, el uso de modelos permite una comunicación más estructurada de los resultados obtenidos, lo que facilita su entendimiento tanto de los investigadores, como los múltiples actores implicados en la administración de los destinos, es así como en este contexto los encuestados manifestaron lo siguiente:

\section{Tabla 3}

¿Considera que un modelo de gestión es necesario para el turismo?

\begin{tabular}{lll}
\hline \multicolumn{1}{c}{ Tipo } & \multicolumn{1}{c}{ Frecuencia } & \multicolumn{1}{c}{ Porcentaje válido } \\
\hline Promotores & 282 & $73,52 \%$ \\
\hline Pasivos & 79 & $20,55 \%$ \\
\hline Detractores & 23 & $5,94 \%$ \\
\hline TOTAL & 383 & $100 \%$
\end{tabular}

Fuente: Elaboración propia

Análisis e interpretación de la tabla 3:

El 73\% de los encuestados le dan total importancia a un modelo de gestión, rango que va del 9 al 10, 21\% pasivos con un rango del 8 al 7 y un 6\% detractores con un rango del 6 al 5 . 


\section{Aspecto turístico}

Por lo que refiere a la índole turística, se considera que existen varios motivos por los cuales el turista tiene una necesidad, un impulso de viajar y actúa en efecto, así por ejemplo un individuo puede viajar por ocio, para descansar, para salir de la rutina, para conocer otras culturas, para degustar nuevas formas de gastronomía, para asistir a algún evento, entre otros. Ahora, muchas veces no tienen consciencia de la motivación por la cual se opta o se rechaza un destino en particular (Beltrán \& Parra, 2017). De ahí que el $53 \%$ de los encuestados concluye que el mayor motivo del viaje es vacaciones, recreo y ocio, el $20 \%$ por visitas a familiares y amigos, compras, educación y formación.

La mayoría de los encuestados, exactamente un 38\% viaja 1 vez cada 6 meses. En otras ciudades como Guayaquil, los resultados arrojan que el 54,67\% de las personas encuestadas respondieron que es muy frecuente la regularidad con la que visitan los sitios turísticos y culturales de Guayaquil. El 36, 67\% lo hace poco frecuente, y el 8, 67\% nada frecuente (Luisa et al., 2017).

En conjunto, el 45 y $42 \%$ de los encuestados viajan de 2 a 6 personas respectivamente. El $60 \%$ de los encuestados viaja de 2 a 3 días y sólo un 6\% no pernocta. El $81 \%$ de los encuestados sí conoce las parroquias rurales de Riobamba, sin embargo, un 19\% no conoce. Las 3 parroquias más conocidas por los encuestados son Cacha, Licán y San Luis, con un $12 \%$ respectivamente. El $49 \%$ de los encuestados sí conoce qué es el TRC y la mitad más uno, es decir $51 \%$ no conoce.

Así pues, el turismo rural comunitario es actualmente una gran oportunidad de desarrollo para las comunidades indígenas de escasos recursos ubicadas en zonas de alto potencial turístico, ya que les brinda la oportunidad de acceder a los beneficios que ofrecen las actividades económicas relacionadas con el turismo. Según la Organización Mundial del Turismo, los países desarrollados y en proceso de desarrollo sumaron más de 470 millones de arribos internacionales el año pasado, de modo que la actividad turística se ha convertido en uno de los principales medios de generación de réditos económicos y empleos para estas economías (Fundación CODESPA, 2011), obteniendo los siguientes resultados, como se muestra en la siguiente tabla 4.

\section{Tabla 4}

¿Le gustaría realizar turismo en las parroquias rurales de Riobamba?

\begin{tabular}{|c|c|c|}
\hline Tipo & Frecuencia & Porcentaje válido \\
\hline Sí & 357 & $93,15 \%$ \\
\hline No & 26 & $6,85 \%$ \\
\hline TOTAL & 383 & $100 \%$ \\
\hline
\end{tabular}


Análisis e interpretación de la tabla 4:

El 93\% de los encuestados no les gustaría realizar TRC en las parroquias de Riobamba.

\section{Tabla 5}

¿Conoce Ud. alguno de estos sitios turísticos?

\begin{tabular}{lll}
\hline \multicolumn{1}{c}{ Centro de TRC } & \multicolumn{1}{c}{ Frecuencia } & \multicolumn{1}{c}{ Porcentaje válido } \\
\hline Palacio Real & 103 & $11 \%$ \\
\hline Jatari Campesino & 37 & $4 \%$ \\
\hline La Moya & 82 & $9 \%$ \\
\hline Quilla Pacari & 21 & $2 \%$ \\
\hline Chakana & 26 & $3 \%$ \\
\hline Casa Cóndor & 80 & $8 \%$ \\
\hline Tambo Huasha & 24 & $2 \%$ \\
\hline Pucará Tambo & 89 & $9 \%$ \\
\hline Ninguno & 201 & $52 \%$ \\
\hline TOTAL & 663 & $100 \%$ \\
\hline
\end{tabular}

Fuente: Elaboración propia

Análisis e interpretación de la tabla 5:

El 52\% de los encuestados no conoce ninguno de los 8 centros de turismo rural comunitario y Palacio Real es el centro más conocido con el $11 \%$.

\section{Aspecto de marketing}

Por lo que refiere al marketing, considerando el alto valor que ha conseguido la calidad en los productos y servicios en los últimos tiempos, hoy en día se busca ampliar la dimensión de excelencia para los consumidores en cada eje de la oferta (Ibarra \& Casas, 2015), es de esta manera que ahora para alcanzar dicha calidad es importantísimo que el mix de marketing: el producto, el precio, el canal de distribución y la promoción sean óptimos en conjunto.

Sobre esta base, el $44 \%$ de los encuestados contrata más el servicio de alimentos y bebidas cuando viaja. La mayoría de los encuestados cuando viaja realiza actividades de gastronomía típica y fotografía rural e intercambio cultural con un 21, 15 y 12\%. El 60\% de los encuestados nunca contratan servicios de mediadores turísticos como agencia de viajes, el $38 \%$ a veces y el $2 \%$ siempre. Si bien, los canales directos entre los servicios turísticos y consumidores permiten agilizar procesos y atender con inmediatez sus necesidades, amenaza la existencia de los mediadores en el sistema turístico, como son las agencias y operadores de viajes. Hoy por hoy estos modelos de negocio afrontan el riesgo de desintermediación en el turismo, tanto por la competencia directa de los nuevos intermediarios online como Expedia, TripAdvisor (Miranda \& Valencia, 2018). 
Por lo que refiere al promedio de gasto personal, se obtuvieron los siguientes resultados:

\section{Tabla 6}

Cuando viaja, ¿Cuál es el promedio de gasto personal por cada día?

\begin{tabular}{lll}
\hline Promedio & Frecuencia & Porcentaje válido \\
\hline$\$ 25-50$ & 191 & 49,77 \\
\hline$\$ 50-100$ & 149 & 38,81 \\
\hline$\$ 100-200$ & 37 & 9,59 \\
\hline Más de 200 & 7 & 1,83 \\
\hline TOTAL & 383 & $100 \%$ \\
\multicolumn{2}{c}{ Fuente: Elaboración propia }
\end{tabular}

Análisis e interpretación de la tabla 6:

El 50\% de los encuestados gasta al día entre \$25 y \$50 al día en el viaje, seguido del entre $\$ 50$ y $\$ 100$.

Con los recientes avances de la comunicación, marcado por la llegada del internet, el aumento de la conectividad e interactividad en la sociedad ha hecho que surjan nuevas herramientas para la recolección, sistematización y difusión de datos, promoviendo la economía y el conocimiento interactivo y democrático (SEGITTUR, 2015), es así como el medio de comunicación más utilizado por parte de los encuestados es el siguiente:

\section{Tabla 7}

¿Qué medio de comunicación utiliza para obtener información turística?

\begin{tabular}{lll}
\hline \multicolumn{1}{c}{ Medio de comunicación } & Frecuencia & \multicolumn{1}{c}{ Porcentaje válido } \\
\hline Internet & 357 & 49,76 \\
\hline Medios audiovisuales & 44 & 6,10 \\
\hline Medios escritos & 26 & 3,66 \\
\hline Agencia de viaje & 37 & 5,12 \\
\hline Redes sociales & 224 & 31,22 \\
\hline iTour & 30 & 4,15 \\
\hline TOTAL & 717 & $100 \%$ \\
\hline
\end{tabular}

Fuente: Elaboración propia

Análisis e interpretación de la tabla 7:

El 50\% de los encuestados utiliza el internet como la herramienta favorita para solicitar información turística.

En particular, las redes sociales, son un canal que facilita el que la marca se posicione entre los consumidores; actualmente todo se hace por medio de internet: páginas web, redes sociales, correo electrónico, aplicaciones, lo que otorga, a cualquier lugar y 
momento, estar más cerca de sus clientes y registrar que es lo que necesitan y desean. El uso correcto de estas herramientas generarían un gran impacto positivo sobre la empresa (Martínez-Oviedo \& Reynoso-Ibarra, 2016).

En cuanto a la red social más utilizada las personas encuestadas manifiestan que es WhatsApp, seguida por Facebook por la facilidad de comunicación a través de teléfonos inteligentes preferentemente (Martínez-Oviedo \& Reynoso-Ibarra, 2016).

en este contexto, la red social que más ocupan los encuestados es:

\section{Tabla 8}

¿Cuál es la red social que más ocupa?

\begin{tabular}{lll}
\hline \multicolumn{1}{c}{ Tipo } & \multicolumn{1}{c}{ Frecuencia } & \multicolumn{1}{c}{ Porcentaje válido } \\
\hline Facebook & 282 & 36,10 \\
\hline Instagram & 184 & 23,54 \\
\hline WhatsApp & 268 & 34,30 \\
\hline Twitter & 28 & 3,59 \\
\hline Pinterest & 19 & 2,47 \\
\hline TOTAL & 780 & $100 \%$ \\
\hline
\end{tabular}

Fuente: Elaboración propia

Análisis e interpretación de la tabla 8:

El 36\% de los encuestados ocupa la plataforma Facebook, seguido de WhatsApp con el $34 \%$.

Análisis de la oferta

Patrimonio tangible e intangible

\section{Tabla 9}

Resumen del patrimonio tangible e intangible

\begin{tabular}{clll}
\hline Parroquia & \multicolumn{1}{c}{ TRC } & Patrimonio Tangible & \multicolumn{1}{c}{ Patrimonio Intangible } \\
\hline San Juan & - Casa Cóndor & - Bosque Polylepis & - Fiestas en honor a San Juan \\
& - Chakana & - Nevado & Bautista \\
& - Tambo Huasha & - Chimborazo & - Parroquialización \\
& & - Templo Machay & - Paseo procesional del chagra \\
& & - Cañón & - Fiestas de carnaval \\
\hline Calpi & - Jatari Campesino & - Iglesia de Calpi & - Fiestas de Santiago de Calpi \\
& - La Moya & - Iglesia Colonial & - Fiestas en honor a San \\
& - Palacio Real & - Mirador & Agustín \\
& - Quilla Pacari & de Telempala & - Semana Santa \\
& & - Mirador Mira Loma & \\
& & - Piedra Endiablada & \\
\hline
\end{tabular}




\section{Tabla 9}

Resumen del patrimonio tangible e intangible (continuación)

\begin{tabular}{llll}
\hline Cacha & Pucara Tambo & - Inti Raymi & \\
& & - Pawka Raymi & \\
& & - Kapac Raymi & \\
& & - Elección de & la \\
& - Textilería & Kachapak Nusta & \\
\hline
\end{tabular}

Fuente: Elaboración propia

\section{Conclusiones}

Se logró construir el marco teórico referencial a partir de un análisis crítico en temáticas vinculadas con el turismo rural comunitario y la dinámica del marketing. Sobre la base de este marco, siendo el turismo rural una actividad que se desarrolla como parte de una actividad comunitaria, es necesario para su ejecución en primera instancia el apoyo gubernamental y el compromiso de participación de las familias o de la comunidad.

- Al identificar las herramientas y procedimientos metodológicas para la creación del modelo de gestión de marketing del turismo rural comunitario, fue necesario considerar que, de las 11 parroquias rurales de Riobamba, sólo Cacha, Calpi y San Juan cuentan con TRC, además de la exigua participación de los CTC, ya que de los 8 existentes, solo Jatari Campesino, Pucará Tambo y Tambo Huasha participaron activamente en esta investigación.

- La mejor estrategia de marketing es la gestión de una marca con el ideal de hacerla conocida, apetecida y con la concepción positiva en la mente y el corazón de los compradores, esto implica, tomar acciones que creen una conexión con el público para influir en su decisión de elegir destino turístico las parroquias rurales de Riobamba.

- Al no existir un modelo de gestión de marketing para el turismo rural comunitario de Riobamba, esta investigación cumple un papel trascendental ya que servirá de insumo para la elaboración de manuales de funcionamiento, así como el uso de los Gobiernos Parroquiales Rurales en el Ecuador en concordancia con el Código Orgánico de Organización Territorial (COOTAD).

\section{Referencias Bibliográficas}

Alcívar, I. (2020). Turismo y desarrollo rural. Realidades diversas y propuestas sostenibles desde América Latina. En Roles de género del turismo rural de Ecuador; el caso de la provincia de Manabí. http://www.munayi.uleam.edu.ec/turismo-ydesarrollo-rural/ 
Beltrán, M. Á., \& Parra, M. C. (2017). Motivaciones Para Viajar. Cuadernos de turismo, $39,41-65$.

Cabanilla, E., Lastra Bravo, X. B., \& Pazmiño, J. (2017). El turismo doméstico ¿Demanda potencial de los centros de turismo comunitario en Ecuador? PASOS Revista de turismo $y \quad$ patrimonio cultural, $15(3), \quad$ 579-590. https://doi.org/10.25145/j.pasos.2017.15.039

Cabanilla, E. y C. G. (2018). Enrique Cabanilla Vásconez - Carlos Garrido-Cornejo (Número Septiembre).

Cañada, E. (2015). La Comercialización Internacional del Turismo Comunitario. La Experiencia en América Latina. ARA: Revista de Investigación en Turismo, 5(2), 33-47.

CELTA. (2018). Octubre, 2018. Tubos y accesorios CELTA su aliado, 1-29.

Chan, W. Y., To, C. K. Man, \& Chu, W. C. (2016). Desire for experiential travel, avoidance of rituality and social esteem: An empirical study of consumer response to tourism innovation. Journal of Innovation and Knowledge. https://doi.org/10.1016/j.jik.2016.02.001

Centro Iberoamericano de Desarrollo Estratégico Urbano [CIDEU]. (2020). Centro Iberoamericano de Desarrollo Estratégico Urbano. Obtenido de https://www.cideu.org/miembro/riobamba/

Doumet, N., Félix, G. \& Huerta, J. (2017). Estrategias para la comercialización de un centro de turismo comunitario Marketing strategies for a tourism community center. Kalpana, 17-29.

Fundación CODESPA. (2011). Modelo de gestión del turismo rural comunitario de CODESPA UNA EXPERIENCIA REGIONAL ANDINA. http://www.bibliotecavirtual.info/wp-content/uploads/2013/02/modelo-gestionturismo-rural-comunitario-codespa.pdf\%0Afile:///C:/Users/77122172K/Downloads/modelo-gestion-turismo-rural-comunitario.pdf

García R., N. (2017). Modelo de Gestión para Fomentar el Desarrollo Turístico de las Comunidades Manabitas, Ecuador. CULTUR: Revista de Cultura e Turismo, 11(2), $81-108$.

García Reinoso, N. C. (2020). La validación del Modelo de Gestión sostenible para el desarrollo turístico en vinculación Universidad. Obtenido de Comunidades Manabitas Ecuador. Investigación \& Negocios: https://doi.org/10.38147/invneg.v13i21.82 
Guillén, S. (2016). Vadece, un modelo para la gestión de marketing, para potenciar el turismo rural y comunitario. Revista de Marketing Aplicado, 1(017), 85-103. Obtenido de https://doi.org/10.17979/redma.2016.01.017.4865

Guillén Herrera, S. R., \& Vera Peña, V. M. (2020). Impacto de la Tecno Ciencia en la Gestión del Marketing del Turismo Comunitario. 20-26.

Hoyos, R. (2016). La Gestión Integral De Un Gerente. 226. https://books.google.com.pe/books?id=oMC4DQAAQBAJ

Ibarra, L. E., \& Casas, E. V. (2015). Aplicación del modelo Servperf en los centros de atención Telcel, Hermosillo: una medición de la calidad en el servicio. Obtenido de Contaduría y Administración, 60(1), 229-260.

López, B. J., López, B. L., \& Peña, V. J. (2015). (2015). Sistemas de Información de Marketing: Una Visión integradora. Tourism \& Management Studies, v.11, n.2, pp.197-203. Obtenido de http://www.redalyc.org/articulo.oa?id=388743884023

Lucas-Marcillo, S. M. S. (2019). Turismo sustentable, tendencia emergente para la economía local con el marketing 2.0 para su impulso - Dialnet. 5, 148-170. https://dialnet.unirioja.es/servlet/articulo?codigo=7152627

Luisa, A., Brunett, L., Humberto, A., \& Brunett, L. (2017). Turismo 2. 0 como herramienta para promocionar los atractivos culturales de Guayaquil Tourism 2. 0 as a tool to promote the cultural attractions of Guayaquil. Innova, 2(6), 154-163. file://C:/Users/USER/Downloads/299-Texto del artículo-877-5-10-20190530 (1).pdf

Martínez-Oviedo, A. F., \& Reynoso-Ibarra, O. Y. (2016). Revista de Negocios \& Pymes. Revista de Negocios \& Pymes, 2, 34-44. https://ecorfan.org/spain/researchjournals/Negocios_y_PyMES/vol2num5/Revista_ de_Negocios_\&_PYMES_V2_N5.pdf\#page=41

Martínez, A., Eva, F., Amboage, S., Sánchez, P. M., \& Viñán-Merecí, C. (2018). Informe técnico sobre el turismo rural comunitario como instrumento para el desarrollo humano a editores: Valentín Alejandro Martínez Fernández Eva Sánchez amboage $m^{a}$ m.... December.

Ministerio de Turismo [MINTUR]. (2020). Rendición de cuentas 2020. Obtenido de Contribución directa del turismo al PIB: chromeextension://efaidnbmnnnibpcajpcglclefindmkaj/viewer.html?pdfurl=https\%3A\% 2F\%2Fwww.turismo.gob.ec\%2Fwpcontent $\% 2$ Fuploads $\% 2 F 2021 \% 2 F 05 \% 2$ FPresentacio $\% 25 C C \% 2581$-Informe- 
de-Rendicio\%25CC\%2581n-de-Cuentas-MINTUR-2020-

compressed.pdf $\&$ clen $=6043864 \&$ chunk $=$ tru

Miranda, V., \& Valencia, A. (2018). La supervivencia de las Agencias de viaje y Turismo: Gestión del Marketing Digital como estrategia para la atracción de clientes turistas. Pontificia Universidad Católica Del Perú Facultad De Gestión Y Alta Dirección, 163.

http://tesis.pucp.edu.pe/repositorio/bitstream/handle/20.500.12404/13122/MIRAN

DA_VALENCIA_LA_SUPERVIVENCIA_DE_LAS_AGENCIAS_DE_VIAJE_ Y_TURISMO_GESTION_DEL_MARKETING_DIGITAL.pdf?sequence=1\&isAl lowed=y

Mogrovejo Lazo, A. E., Luna Altamirano, K. A., Ormaza Andrade, J. E., Castro Vázquez, P. G., \& Torres Beltrán, A. M. (2019). Plan de marketing en el sector salud. Ciudad de Cuenca, Ecuador. 3, 1-111.

Organización de las Naciones Unidas para la Educación, la Ciencia y la Cultura [UNESCO]. (2021). Comisión Nacional Ecuatoriana de Cooperación con la UNESCO. Obtenido de https://es.unesco.org/countries/ecuador

Organización Mundial de Turismo [OMT]. (10 de 03 de 2021). Tablero de datos del turismo. Obtenido de https://unwto.org.es

Parra Cárdenas, A. V., Cisneros Mustelier, L., \& Velasteguí López, E. (2019). El turismo rural y aportaciones al desarrollo de comunidades en territorios locales. Explorador Digital, 3(4), 6-28. https://doi.org/10.33262/exploradordigital.v3i4.912

Patiño Paucar, A. (2016). Turismo rural comunitario como estrategia de desarrollo local para el municipio de Mejía.

Pearce, D. (2016). Modelos de gestión de destinos. Estudios y Perspectivas en Turismo, 25(1), 1-16.

Ramírez, M., \& Perusquia, J. (2019). El Sistema de Información de Marketing como modelo de gestión basado en la comunicación organizacional. Espacios, 40(0798 1015), 30. http://www.revistaespacios.com/a19v40n27/a19v40n27p30.pdf

Reyes, M. V., Ortega, Á. F., \& Machado, E. L. (2017). Modelo Para La Gestión Integrada Del Turismo. REVESCO. Revista de Estudios Cooperativos.

Rodas, M., Ulluari, N., \& Sanmartín, I. (2015). El Turismo Comunitario en el Ecuador: Una revisión de la literatura. Revista de Turismo, 9(9), 60-77. file:///C:/Users/UNEMI.24-H102.000/Desktop/investigacion/19-Texto del artículo104-1-10-20190116 (1).pdf 
Saltos Cruz, J. G., Carvajal Larenas, R. P., \& Yessenia Elizabeth, C. M. (2018). Marketing social para el sector industrial de Tungurahua, un estudio causal a partir de análisis factorial. 2, 1-9.

Santamaría, G. E. R., Vintimilla, M. C. Z., Romero, G. N. F., Vizuete, D. D. C., \& Báez, L. A. Q. (2017). La Potencialidad Turística En Los Proyectos De Turismo Rural Comunitario. European Scientific Journal, ESJ, 13(20), 219. https://doi.org/10.19044/esj.2017.v13n20p219

SEGITTUR. (2015). Smart destination. Informe destinos turísticos inteligentes: construyendo el futuro. Madrid.

Torres, M. (2016). Desarrollo de un Modelo de Gestión para potenciar el Sector Turístico Comunitario Rural Departamento de Investigación y Postgrados abril 2016.

Vaca Rodríguez, G. L. (2015). Estudio de Factibilidad para la creación de una microempresa asociativa enfocada al Turismo Rural, en la Provincia de Tungurahua. 3(2), 54-67. http://repositorio.unan.edu.ni/2986/1/5624.pdf

Valdiviezo Medina, B. I. (2015). Trabajo de titulación.

Vera, I. A. (2018). Realidades del Turismo Local Compilación de Opiniones Turísticas. http://library1.nida.ac.th/termpaper6/sd/2554/19755.pdf

Vera Páez, K. V. (2017). Escuela De Administración De Empresas.

\section{Ciencia}




\section{VPDigital}

El artículo que se publica es de exclusiva responsabilidad de los autores y no necesariamente reflejan el pensamiento de la Revista Visionario Digital.

\section{LCiencia}

El artículo queda en propiedad de la revista y, por tanto, su publicación parcial y/o total en otro medio tiene que ser autorizado por el director de la Revista Visionario Digital.
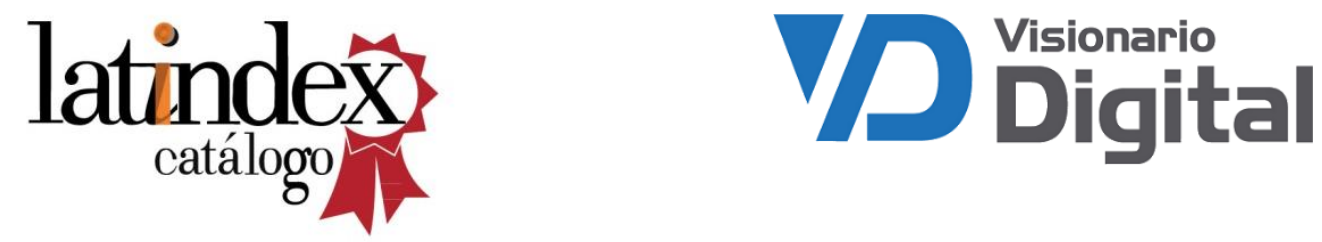

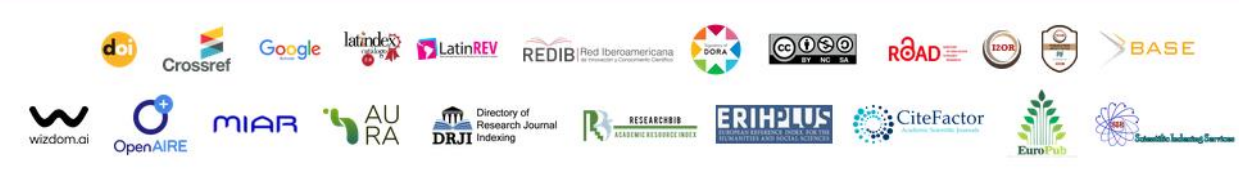

